\title{
The Key to Employee Motivation A Competitive Leadership Approach
}

\author{
Katharina Rebecca Tesmer ${ }^{1}$ and Carolyn Oxenford ${ }^{1}$ \\ ${ }^{1}$ Marymount University, Arlington, Virginia, United States \\ DOI: https://doi.org/10.47611/jsr.v9i2.808
}

\section{ABSTRACT}

Capitalism has created a highly competitive corporate culture in the United States in which organizations must strive to increase their competitive advantage and maximize financial performance. To achieve these long-term goals, business leaders must keep employees innovative, engaged and motivated. However, current efforts to increase employee engagement often fail and even deplete employee engagement (Pink, 2009). Successful leaders need to be open to new approaches that have proven to be effective (Pink, 2009). This paper focuses on the importance of psychological approaches to employee motivation as a path to increased functionality and success of any organization.

\section{What is Motivation?}

Leaders want their employees to show interest and feel related to the organization and its goals. Hence, most leaders focus on maximizing employee engagement. However, employee engagement is only a part of the bigger concept that determines employee performance. The wholistic concept that entails all necessary employee variables is employee motivation. Employee motivation includes the employees' sense of relatedness (often referred to as engagement), competence and autonomy, all of which impact productivity and performance. Employee motivation is an essential predictor of success as it positively correlates with effort, social connectivity, creativity, job satisfaction and retention rates (Linley \& Harrington, 2010). Furthermore, motivated employees exhibit a higher sense of accountability and responsibility (Linley \& Harrington, $2010 \&$ Kouzes \& Posner, 2017). All of these outcomes benefit the overall functioning and success of any organization. Hence, leaders have the opportunity to optimize the business outcomes of their organization by increasing employee motivation.

\section{Extrinsic and Intrinsic Motivation}

Before business leaders can maximize employee motivation through specific practices, they must first understand the different types of motivation. Pink (2009) explains that human motivation may be characterized as extrinsic and intrinsic. Extrinsic motivation is based on external rewards (usually material) that are given as a result of specific behaviors. For instance, if an employee completes a task because he or she will receive a commission, he or she is 
extrinsically motivated. On the other hand, intrinsic motivation originates from the individual, which means that employees are motivated by the satisfaction that comes from engaging in an activity. For instance, an employee might feel intrinsically motivated to design a poster because he or she enjoys that type of work. Pink (2009) describes intrinsic motivation as the most powerful human drive because it leads employees to pursue mastery. Even when a goal is reached, intrinsically motivated employees will continue to pursue the activity due to the satisfaction that derives from it. This persistence leads to improvements and innovations at the workplace, which is highly beneficial to the overall functioning and success of any organization. Employees who are intrinsically motivated display more interest, creativity, persistence and enhanced performance (Linley \& Harrington, 2010), which are vital characteristics that elevate productivity.

\section{Current Engagement Efforts}

Currently, most leaders target extrinsic motivation, hoping to optimize employee performance. Leaders give benefits, bonuses and other financial rewards to recognize and encourage good behavior. Kouzes and Posner (2017) call these "if-then" rewards because employees know "if" they achieve the goal, "then" they get the reward. This simple motivation method seems functional and logical, but it has three major flaws: a) behavior is unlikely to exceed expectations (Pink, 2009); b) unethical and unlawful behaviors may be fostered (Kouzes \& Posner, 2017); and c) intrinsic motivation diminishes (Pink, 2009).

Employee behavior that does not exceed expectations may not seem problematic at first glance. However, if employees only reach expected goals, they are less likely to demonstrate innovative or creative behavior (Pink, 2009). Employees would only do the minimum to receive their material reward (Pink, 2009). Organizations will have a hard time improving processes and gaining the competitive edge without innovations (Pink, 2009).

Fostering extrinsic motivation encourages employees to seek the quickest way possible to gain that reward regardless of any consequences. This focus on the end goal is more likely to lead to unethical and unlawful behavior because external rewards don't emphasize the process, they only emphasize the outcome (Kouzes \& Posner, 2017).

Lastly, external rewards are problematic because they deplete intrinsic motivation (Pink, 2009), the most powerful human drive. When external rewards are given after a project is completed, the employee does not complete the project for him- or herself. The employee completes the task because he or she seeks to receive the reward. Over time, the performance of the employee becomes depended on the external reward while the employee's intrinsic motivation diminishes. Now, the employee will have a hard time completing the project if the reward is not given after because his or her intrinsic motivation has been depleted. As stated earlier, intrinsic motivation enables creativity and innovation which are both essential to reach a competitive edge in the current business environment. That is why depleting intrinsic motivation of employees would be detrimental to the success and competitive advantage of any organization. Therefore, leaders should seek to maximize intrinsic motivation.

\section{Understanding Human Motivation at Work: Self Determination Theory}

Human motivation can best be understood Deci and Ryan's self-determination theory (2000). According this theory, individuals seek to meet the primary psychological needs of relatedness (referred to earlier as engagement), 
competence and autonomy (Deci \& Ryan, 2000). As Pink states, "when those needs are satisfied, we're motivated, productive and happy. When they're thwarted, our motivation, productivity, and happiness plummet" (2009, p.70). Hence, leaders can optimize employee performance by facilitating employees' senses of relatedness, competence and autonomy using scientifically successful leadership practices.

To foster relatedness in employees, leaders should develop a meaningful purpose and shared values; model behavior, show involvement and build relationships. By setting goals as well as recognizing and challenging employees, leaders develop competence in their employees. Lastly, leaders foster autonomy by empowering their employees.

\section{Relatedness}

For employees to be motivated at their workplace, they need to feel related to the purpose and values of the organization; to their leader; and to their coworkers. Employees feel the need to belong, to be part of something that they believe in. This starts with the bigger purpose and the core values of the organization. The impact of a purpose depends on its distinctiveness, emotional impact and connectiveness to the organization's guidelines. Interestingly, the distinctiveness of the purpose positively relates to its effectiveness (Kouzes \& Posner, 2017). Hence, the more unique the purpose of an organization is, the higher will be the positive impact of that purpose (Kouzes \& Posner, 2017). Furthermore, if leaders convey the purpose in a passionate manner then it is likely to unleash positive emotions of enthusiasm and hope (Kouzes \& Posner, 2017). These emotions foster commitment to the organization as well as motivation (Kouzes \& Posner, 2017). Connecting the broader purpose to guidelines, goals and daily tasks positively affects employee performance, health, engagement and the financial performance of the organization (Kouzes \& Posner, 2017). Overall, a meaningful purpose has proven to establish higher employee commitment and trust in management and relationships (Linley \& Harrington, 2010).

Building on the motivation that derives from a meaningful purpose, identifying and imbedding shared values also raises employee motivation. Fortunately, many leaders already realize the need for company values and have made it their goal to maximize social impact by supporting communities, charities, etc. (Linley \& Harrington, 2010 $\&$ Pink, 2009). However, these company values were mostly established by the leaders and not in communication with their employees. Regular, open communication about values is essential because "a unified voice on values results from discovery and dialogue" (Kouzes \& Posner, 2017, p.63). At first leaders should identify their own values and then inquire about their employees' values (Kouzes \& Posner, 2017). That way all employees are included in the conversation and are more likely to believe in and represent these shared values (Kouzes \& Posner, 2017). Once shared values are identified and manifested, employee commitment, loyalty, attitude, motivation and feeling of personal effectiveness are optimized (Kouzes \& Posner, 2017). Imbedding shared values into everyday processes is essential, and it starts with the leader. Acting based on one's values is beneficial to leaders themselves because it increases their self-confidence, decreases stress and makes it much easier to make decisions (Kouzes \& Posner, 2017).

In addition, if the leader represents the shared values, then employees are more likely to trust their leader and see him or her as a role model. Conveying shared values through passionate first-person stories as a leader is most effective to motivate employees (Kouzes \& Posner, 2017). Hence, acting based on shared values benefits the leader because connection to the employees is strengthened. Overall, a meaningful purpose and shared values lay the foundation for employee motivation. Deriving guidelines, goals and a vision from shared values elevates employee 
motivation because when personal values align with goals - self-concordance - then employee effort and well-being increase (Linley \& Harrington, 2010).

Role-modeling is an essential part of successful leadership because employees reflect the actions of their leader. Therefore, leaders should act based on purpose and shared values (Kouzes \& Posner, 2017), display positive emotions (Kouzes \& Posner, 2017) and seek and accept feedback (Kouzes \& Posner, 2017 \& Linley \& Harrington, 2010). Reflecting the purpose and shared values as a leader also establishes trust because employees see that their leader "practices what he/she preaches" (Kouzes \& Posner, 2017, p. 43). Furthermore, showing positive emotions of passion and enthusiasm about the organization and its goals will motivate others (Kouzes \& Posner, 2017). Positive emotions boost work morale because employees are unlikely to get excited about the organization and its goals if their leader isn't even excited about it (Kouzes \& Posner, 2017).

Leaders should also seek feedback and accept it when it is offered. This does not only signal that he or she cares and seeks to grow, but also establishes trust. Asking for and accepting feedback also makes employees more likely to accept the leader's feedback in the future (Kouzes \& Posner, 2017)

Aside from representing key behaviors, leaders also have to be involved within their organization to foster relatedness. A big part of being involved is simply showing up to meetings and events because physical presence shows employees that their leader cares about them and values their contributions to the organization (Kouzes \& Posner, 2017). Face-to-face interactions have been shown to build the strongest interpersonal connections (Kouzes \& Posner, 2017). Aside from building valuable relationships with employees, leaders also learn about everyday processes within the organization which helps them to make business decisions. This includes not only coming to meetings and engaging with employees regularly, but also talking to other departments and closely following what competitors are doing. Leaders always have to be aware of changes and new opportunities. Feedback also plays a big role in the leader's involvement (Kouzes \& Posner, 2017). As mentioned earlier, seeking and accepting feedback sets the tone for employees' attitude on feedback. Feedback is most effective when it is given right after the action and when it is authentic (Kouzes \& Posner, 2017). For feedback to be authentic a leader should be honest and direct (Kouzes \& Posner, 2017).

Lastly, for employees to feel socially related at work, leaders need to create the conditions for relationships to flourish. A positive mindset at the workplace starts with appreciative inquiring, which means that leaders ask positive questions about the organization (Linley \& Harrington, 2010). For instance, they might ask; What is working well or what can we learn from other departments? (Linley \& Harrington, 2010). Positive psychology entails the idea of highlighting the strengths of the organization because "to foster team spirit, build optimism, promote resilience, and renew faith and confidence, leaders look at the bright side. They keep hope alive" (Kouzes \& Posner, 2017, p.134). Asking positive questions changes the conversation and fosters positive emotions of hope, enthusiasm and excitement (Linley \& Harrington, 2010). Positive emotions bring employees together because they promote motivation, commitment and social connections (Linley \& Harrington, 2010). When leaders set a positive tone, they foster a supportive culture instead of a competitive culture because employees start to engage more with one another (Linley \& Harrington, 2010). Specifically, when employees are driven by positive emotions then they are more likely to help others (Linley and Harrington, 2010). These acts of altruism generate further positive emotions within the individual (Linley \& Harrington, 2010). Additionally, the employee who is being helped feels grateful for the help which leads to the desire to give back (Linley \& Harrington, 2010). This chain reaction of positive emotions moves through the entire 
organization and mobilizes skills and expertise that were only available to the individual at first (Linley \& Harrington, 2010). This interpersonal engagement increases trust which further strengthens the social connections at work (Linley \& Harrington, 2010). Overall, positive emotions lead employees to feel related to the organization, coworkers and their leader, which is also referred to as relational strength (Linley \& Harrington, 2010).

\section{Competence}

According to self-determination theory, every individual has the psychological need to feel competent. Leaders have the opportunity to foster the feeling of competence by giving recognition, providing training, offering challenges and setting goals.

One of the ways for leaders to elevate employees' feeling of competence is by personally recognizing them. The field of positive psychology encompasses various effective recognition strategies because recognizing others unleashes positive emotions (Linley \& Harrington, 2010). Effective recognition is specific, public and appreciative (Kouzes \& Posner, 2017).

Specific and personal recognitions are most powerful (Kouzes \& Posner, 2017). To make specific recognitions leaders need to be involved and know their employees. Another effective way of recognizing employees is through events and public announcements (Kouzes \& Posner, 2017). Praising employees in front of their coworkers positively impacts their work morale because it gives guidelines of what is expected of employees, establishes commitment of all and makes the individual feel valued (Kouzes \& Posner, 2017).

Simple expressions of gratitude also foster employees' feeling of competence because it shows them that their leader respects and appreciates their work. Being grateful also benefits the leader because people who show gratitude maintain higher levels of well-being (Kouzes \& Posner, 2017). To ensure that employees repeatedly express desired behavior, precise feedback should be given as to why their behavior was great (Kouzes \& Posner, 2017).

The positive emotions that are generated by effective recognition have a significant impact on the performance of the employees. Positive emotions have shown to promote resilience, perseverance, creativity, innovation, knowledge, health, flexibility, optimal functioning, achievement and motivation (Linley \& Harrington, 2010 \& Kouzes \& Posner, 2017). Specifically, positive emotions broaden employees' perspective and attention, resulting in a more open-minded, expansive mindset (Linley \& Harrington, 2010). This broad perspective allows for creativity and innovation at the workplace (Linley \& Harrington, 2010 \& Kouzes \& Posner, 2017). On the other hand, negative emotions narrow focus, which reduces innovation and creativity (Linley \& Harrington, 2010). Hence, a ratio of one negative emotion for every three positive emotions would be ideal to ensure desired level of creativity and innovation (Linley \& Harrington, 2010).

Aside from recognition, challenging employees is an important practice to extend their employees' competencies. Here, it is essential that leaders provide appropriate training and growth opportunities for employees to expand their skillset and confidence. To achieve optimal levels of employee performance and satisfaction, leaders should strive to foster an optimal state of mind, characterized as flow by Csikszentmihalyi (Pink, 2009). Flow can be reached when the skill level of an individual equals the extent of the challenge (Kouzes \& Posner, 2017). Once an individual is absorbed in this state, he or she forgets time and space and becomes highly focused on the activity (Kouzes \& Posner, 2017). When individuals withdraw from flow, they are eager to engage into the activity again due to the high 
levels of satisfaction they experienced as a result of their flow experience. Hence, leaders have the opportunity to maximize their employees' motivation and performance, if they create the optimal conditions for flow states to occur.

Fostering employees' feeling of competence through recognition and challenges concludes with appropriate goal setting. For employees to feel competent, they need to know what they are working toward and whether they are achieving these goals. However, leaders need to differentiate between different types of goals because each type fosters a different kind of motivation. Performance goals foster extrinsic motivation because the focus is the end result, not the path, nor the future (Pink, 2009). Hence, unethical and unlawful behaviors are fostered because only the end result is valued and rewarded (Kouzes \& Posner, 2017). For instance, a performance goal might be a monthly report, or the quantity of customer service given in a day. When working toward performance goals, leaders often encourage their employees to focus on problems, which fosters negative emotions and narrows employees' focus (Linley \& Harrington, 2010). Instead of setting performance goals, leaders should strive to set learning or purpose goals because they increase and maintain intrinsic motivation of employees (Pink, 2009). Compared to performance goals, learning goals are less specific and are targeted toward perfecting a skill such as being proficient in Excel. Flow experiences are more likely to result from learning goals because pursuing the activity itself is the goal instead of the outcome. Setting learning goals benefits the overall functionality and success of the organization because employees push for mastery and display higher task performance (Linley \& Harrington, 2010). Because learning goals are rather abstract and long-term, they may contain smaller goals which are called process goals. Process goals are small goals that make up long-term goals and give employees a step-by-step guideline (Pink, 2009). These process goals elevate employees' work morale because "small wins boost inner work life" (Kouzes \& Posner, 2017, p. 174).

\section{Autonomy}

For employees to reach optimal levels of motivation, they need to feel autonomous and empowered by their leaders. Empowering leadership practices generate higher levels of employee motivation than controlling leadership practices (Linley \& Harrington, 2010). In addition, personal autonomy correlates with happiness, which in turn creates positive emotions (Linley \& Harrington, 2010). Fortunately, autonomy leads to shared accountability and responsibility (Kouzes \& Posner, 2017). Leaders who show modesty and humility further increase employees' feeling of autonomy (Linley \& Harrington, 2010). Surprisingly, "You become more powerful when you give your power away" (Kouzes \& Posner, 2017, p. 221).

A combination of leadership practices empowers employees. Most of these strategies target bidirectional trust because trust eliminates the need of the leader's control (Kouzes \& Posner, 2017). Most importantly, leaders should give their employees autonomy through choices because the possibility to choose increases self-directedness, commitment, motivation and productivity (Kouzes \& Posner, 2017). Aside from providing choices, leaders should also give their employees chances to innovate and take initiative. One way to provide a chance for innovation is by implementing a 20\% culture (Kouzes \& Posner, 2017 \& Pink, 2009). Within a 20\% culture, employees are encouraged to spend one day a week on a project of their choice (Kouzes \& Posner, 2017 \& Pink, 2009). Many companies who have implemented the $20 \%$ culture have developed major process improvements (Kouzes \& Posner, 2017 \& Pink, 2009). 
Another way for leaders to empower their employees is through communication strategies such as asking questions, asking for help; and highlighting strengths. Leaders are an important resource for employees and therefore leaders need to listen actively to their employees instead of only giving instructions (Kouzes \& Posner, 2017 \& Linley \& Harrington, 2010). Leaders should listen to their employees' ideas and concerns and focus on understanding them rather than giving quick responses. Secondly, asking employees for help boosts their feeling of autonomy because it makes them feel valuable and shows that their leader trusts them (Kouzes \& Posner, 2017). Leaders can also empower their employees by highlighting their strengths because it boosts their self-confidence. By identifying employees' strengths, the leader also establishes authenticity which increases trust (Kouzes \& Posner, 2017). Aside from communication strategies, leaders also need to provide resources such as training and removing obstacles to optimize employees' feeling of autonomy (Kouzes \& Posner, 2017).

\section{Conclusion}

Capitalism has created a highly competitive corporate culture in which leaders strive to reach and maintain a competitive advantage. This goal can only be reached and maintained if their workforce is committed to the organization and motivated to give their best. Deci and Ryan's self-determination theory offers a foundation for successful leadership practices that motivate employees by satisfying the three basic psychological needs of relatedness, competence and autonomy. Relatedness can be enhanced by developing a meaningful purpose and shared values; modeling behavior, showing involvement and building relationships. Employees' feeling of competence can be elevated by setting goals, giving recognition and offering challenges. Autonomy can be fostered by offering choices, providing opportunities for innovation and using empowering communication strategies. To the extent that leaders can develop effective strategies in these areas, employees will be more innovative and productive. Employees will also be happier and less likely to leave the organization.

Leaders are vital to setting the tone and establishing the conditions that maximize employee motivation and productivity. While some leaders seem to be aware of the importance of employee engagement (relatedness), employees' senses of competence and autonomy also play an important role toward satisfaction and productivity. Research constantly expands our knowledge on the best leadership practices. Due to this constant learning process, leadership should be seen as a skill that can be acquired over time. Even though some people are naturally gifted to lead, scientific evidence proves that anyone can become a great leader.

\section{References}

Deci, E. and Ryan, R. (2000). Self determination theory and the facilitation of Intrinsic Motivation, Social Development and Well-Being. Retrieved 19 July 2019, from https://selfdeterminationtheory.org/SDT/documents/2000 RyanDeci SDT.pdf

Pink, D. (2009). Drive. New York, NY: Riverhead Books. 
Kouzes, J. and Posner, B. (2017). The leadership challenge. 6th ed. Hoboken, New Jersey: John Wiley \& Sons.

Linley, P. and Harrington, S. (2010). Oxford handbook of positive psychology and work. New York: Oxford University Press, pp.67-117, 155-188, 235-249, 323-333. 